\title{
Short communication: Latin-style fresh cheese enhances lactic acid bacteria survival but not Listeria monocytogenes resistance under in vitro simulated gastrointestinal conditions
}

\author{
C. C. G. Silva, ${ }^{1}$ M. F. P. Domingos-Lopes, V. A. F. Magalhães, D. A. S. R. Freitas, M. C. Coelho, H. J. D. Rosa, \\ and M. L. N. E. Dapkevicius \\ CITA-A, Centro de Investigação e Tecnologias Agrárias dos Açores, Universidade dos Açores, 9700-042 Angra do Heroísmo, Açores, Portugal
}

\begin{abstract}
Different studies in humans have provided evidence about the health benefits of probiotics. However, most probiotic strains do not maintain good viability in the harsh conditions of the gastrointestinal tract (GIT). In the present study, Latin-style fresh cheese produced with potential probiotic bacteria was tested to evaluate this cheese type as a food carrier for the delivery of viable microorganisms after exposure to simulated GIT conditions. The resistance of 28 lactic acid bacteria (LAB) strains and Listeria monocytogenes upon exposure to acidic conditions ( $\mathrm{pH} 2.5$ ) and bile and pancreatic enzymes ( $0.3 \%$ of bile salts and $0.1 \%$ of pancreatin) was evaluated in vitro. When compared with fresh cultures, fresh cheese greatly improved LAB survival to simulated GIT conditions, as no loss of viability was observed in either acidic conditions $(\mathrm{pH} 2.5)$ or bile salts and pancreatin environment over a 3 -h period. In opposition, L. monocytogenes did not survive after $1 \mathrm{~h}$ under acidic conditions. These data demonstrated that Latin-style fresh cheese could play an important role in probiotic protection against gastrointestinal juices, enhancing delivery within the gut and thereby maximizing potential health benefits of LAB.
\end{abstract}

Key words: fresh cheese, lactic acid bacteria, probiotic, Listeria monocytogenes

\section{Short Communication}

Increasing evidence indicates that consumption of some lactic acid bacteria (LAB) strains reduces the risk of various diseases (Fijan, 2014). For this reason, interest in probiotic bacteria as dietary adjuncts has increased and new probiotic strains have been incorporated into numerous foods and beverages (Corbo et al., 2014). Nevertheless, to be effective and confer

Received February 4, 2015.

Accepted March 15, 2015.

${ }^{1}$ Corresponding author: celia@uac.pt their beneficial effect on the host, probiotics must survive passage through the human gastrointestinal tract (GIT) and be present in sufficient numbers to colonize the human small and large intestine (Collins et al., 1998). To establish such a probiotic trait, in vitro simulators of digestion have been used for probiotic strain screening (Charteris et al., 1998; Gardiner et al., 1999; Cook et al., 2012; Burns et al., 2014).

Numerous reports have shown that bacteria from most probiotic products are not acid resistant and do not survive passage through the GIT (Ritter et al., 2009; Burns et al., 2014). Thus, to improve the survival of these bacteria in the human GIT several approaches have been investigated, including encapsulation or physical protection in the food system (Cook et al., 2012). Fermented milks and yogurts have been employed as delivery vehicles of probiotics, but some studies suggest that they may not be ideal food carriers for certain probiotic strains, due to the low $\mathrm{pH}$ and the high oxygen levels in the liquid matrix (Gardiner et al., 1999; Vinderola et al., 2000). In contrast, cheese has certain advantages as it creates a buffer against the highly acidic environment in the GIT. Moreover, the dense matrix and relatively high fat content of cheese may offer additional protection to probiotic bacteria in the stomach, enhancing probiotic survival throughout gastric transit (Sharp et al., 2008; Karimi et al., 2012). Nonetheless, survival of probiotic bacteria in cheese must be ensured, as many probiotic cultures are affected by cheese manufacture protocols and subsequent ripening. Latin-style fresh cheeses are traditionally made in Portugal and Spain from pasteurized goat or cow milk, without addition of a starter culture. They are ready for consumption immediately after production and are characterized by their soft paste and fresh mild flavor. These fresh cheeses may constitute an ideal food carrier for probiotic cultures because their $\mathrm{pH}$ is almost neutral, they have high moisture content, and no addition of starter or adjunct cultures that may compete with potential probiotic bacteria. Additionally, they have a simple manufacturing process, low cost, and good ac- 
ceptance by most consumers due to their mild taste (Evert-Arriagada et al., 2013). However, due to the relatively high $\mathrm{pH}$ and water activity, fresh cheeses are particularly sensitive to colonization by Listeria monocytogenes through postprocess contamination (Kabuki et al., 2004). Thus, the present study was aimed to investigate the effect of this type of fresh cheese on the protection of several LAB strains and a pathogen ( $L$. monocytogenes) to adverse gastrointestinal conditions. Therefore, the resistance of $28 \mathrm{LAB}$ strains and $1 \mathrm{~L}$. monocytogenes strain to low $\mathrm{pH}$, bile salts, and digestive enzymes was evaluated in vitro using both pure cultures and the inoculated cheese samples.

The LAB strains isolated from an artisanal cheese (Pico cheese) were identified by $16 \mathrm{~S}$ rRNA sequencing analysis as indicated by Ribeiro et al. (2014). The 28 studied strains were identified as Lactobacillus paracasei (14), Lactobacillus plantarum (5), Lactobacillus paraplantarum (1), Lactobacillus otakiensis (1), Lactococcus lactis (2), Leuconostoc mesenteroides (4), and Leuconostoc citreum (1). Nucleotide sequence data have been assigned GenBank Accession numbers KF193424, KM079353-KM079358, KM079360, KM079361, KM096813-KM096826, KM096828, and KM103931KM103934. All LAB strains were grown in de Man, Rogosa, Sharpe broth medium (BK089HA, Biokar Diagnostics, Beauvais, France) and stored with 20\% glycerol (vol/vol; VWR, Fontenay-sous-Bois, France) at $-80^{\circ} \mathrm{C}$ until use. Listeria monocytogenes (ATCC 7466) was also used to inoculate cheese to evaluate its resistance to simulated GIT conditions. Listeria monocytogenes kept at $-80^{\circ} \mathrm{C}$ was revitalized and propagated in Nutrient Broth (AES, Bruz, France) for 18 h, at $37^{\circ} \mathrm{C}$, before use.

To evaluate resistance to low $\mathrm{pH}, \mathrm{LAB}$ strains and $L$. monocytogenes from overnight $(18 \mathrm{~h})$ cultures (cultured at $30^{\circ} \mathrm{C}$ for $\mathrm{LAB}$ or $37^{\circ} \mathrm{C}$ for L. monocytogenes) were harvested by centrifugation $\left(10,000 \times \mathrm{g}, 5 \mathrm{~min}, 4^{\circ} \mathrm{C}\right)$, washed twice with PBS buffer ( $\mathrm{pH} 7.2$, Oxoid, Basingstoke, Hampshire, UK), before being resuspended in PBS solution and adjusted to $\mathrm{pH} 2.5$. To simulate the small intestine conditions, bacterial cells were also resuspended in PBS solution ( $\mathrm{pH} 7.2)$, containing $0.3 \%$ (wt/vol) bile salts (Fluka, Buchs, Switzerland) and $0.1 \%$ pancreatin (Sigma-Aldrich, St Louis, MO). Initial inoculum was corresponding to viable counts of approximately $10^{-7} \mathrm{cfu} / \mathrm{mL}$. Resistance to both conditions was assessed in duplicate, in terms of viable colony counts after incubation at $37^{\circ} \mathrm{C}$ for $0,1,2$, and $3 \mathrm{~h}$, in a shaking incubator (Edmund Bühler GmbH, Hechingen, Germany) reflecting the time spent by food in the stomach and the small intestine. An aliquot of $1 \mathrm{~mL}$ was taken at each time point and serially diluted with buffered peptone water (AES). The LAB strains were enumerated in duplicate on de Man, Rogosa, Sharpe agar (Merck, Darmstadt, Germany) after incubation under aerobic conditions at $30^{\circ} \mathrm{C}$ for $72 \mathrm{~h}$. Listeria monocytogenes counts were carried out by plating appropriate dilutions in duplicate on PALCAM-Listeria selective agar containing supplement (Biokar, Beauvais, France), followed by enumeration after incubation at $37^{\circ} \mathrm{C}$ for $48 \mathrm{~h}$.

To evaluate Latin-style fresh cheese as food carrier of bacteria under the GIT conditions, cheeses without addition of starter cultures were made in a laboratory plant, following the procedure described in detail by Coelho et al. (2014). In each trial, pasteurized cow milk was distributed into $1-\mathrm{L}$ vats and warmed at $32^{\circ} \mathrm{C}$ before each LAB culture $(1 \%$, vol/vol) or L. monocytogenes $(1 \%, \mathrm{vol} / \mathrm{vol})$ was individually inoculated. Initial inoculum corresponded to viable counts of approximately $10^{-7} \mathrm{cfu} / \mathrm{mL}$. The milk was left for $20 \mathrm{~min}$ before the addition of rennet (LMF 1/15,000, $0.2 \mathrm{~g} / \mathrm{L}$, Lusocoalho, Lisbon, Portugal), calcium chloride (0.2 $\mathrm{g} / \mathrm{L})$, and $\mathrm{NaCl}(10 \mathrm{~g} / \mathrm{L})$. The curd was cut, molded into small cheeses $(6.5 \mathrm{~cm}$ in diameter), and stored at $4^{\circ} \mathrm{C}$ until being tested. An aliquot $(2 \mathrm{~g})$ of fresh cheese (after 3 to $5 \mathrm{~d}$ of storage at $4^{\circ} \mathrm{C}$ ), containing each LAB strain or Listeria monocytogenes, was added to each test tube containing $10 \mathrm{~mL}$ of acidic PBS ( $\mathrm{pH} 2.5$ ) or $10 \mathrm{~mL}$ of PBS (pH 7.2) containing $0.3 \%$ bile salts and $0.1 \%$ pancreatin. The contents were mixed well with the help of a sterile rod and all tubes were incubated in a shaking incubator at $37^{\circ} \mathrm{C}$. Bacterial survival was determined at $0,1,2$, and $3 \mathrm{~h}$ by removing 1 aliquot ( 1 $\mathrm{mL}$ ) at each time point. Serial dilutions were performed in buffered peptone water and, subsequently, LAB or L. monocytogenes were enumerated as indicated above for pure cultures. Each experiment was conducted in quadruplicate.

Overall, the pure cultures showed poor resistance to gastric acidity in the absence of cheese matrix, as demonstrated by the rapid loss of viability after exposure to $\mathrm{pH} 2.5$ (Figure $1 \mathrm{~A}$ and $\mathrm{B}$ ). After $2 \mathrm{~h}$ of incubation at this low pH, only 13 strains still persisted (classified as resistant; Figure 1A), although with reduced survival rate. Most of strains (15) were classified as sensitive to low $\mathrm{pH}$, as they were not detected after $2 \mathrm{~h}$ of incubation at $\mathrm{pH} 2.5$ (Figure 1B). Seven strains (6 lactobacili and 1 Lactococcus) were undetectable after $1 \mathrm{~h}$ at this low $\mathrm{pH}$. Yet all the strains tested were undetectable $(<1 \log \mathrm{cfu} / \mathrm{mL}$ ) after $3 \mathrm{~h}$ of exposure to $\mathrm{pH} 2.5$ (Figure $1 \mathrm{~A}$ and $\mathrm{B}$ ). These results were not surprising, as susceptibility to gastric acidity can greatly diverge among species and strains. Nevertheless, several studies reported a considerable loss of LAB viability after ex- 
posure to low pH conditions (Ritter et al., 2009; Cook et al., 2012; Monteagudo-Mera et al., 2012; Gregoret et al., 2013; Rehaiem et al., 2014). Some strains have been showed to be highly resistant to low $\mathrm{pH}$ but, in some of these studies, the bacteria were incubated in the presence of culture medium, which aided their survival (Nueno-Palop and Narbad, 2011; Faye et al., 2012). In the present study, the bacteria were washed and there were no compounds to protect the potential probiotic cells and avoid cell death in the presence of low $\mathrm{pH}$ levels. Thus, after $2 \mathrm{~h}$ of exposure to low $\mathrm{pH}$, the cell numbers of most bacteria decreased to values lower than $10^{3} \mathrm{cfu} / \mathrm{mL}$ (Figure $1 \mathrm{~A}$ and $\mathrm{B}$ ), which is below any levels generally accepted for probiotic bacteria to exert a therapeutic effect. Therefore, to exert any probiotic activity these bacteria would require protective agents to survive the passage through the stomach at high numbers. Thus, Latin-style fresh cheeses were prepared with the same LAB cultures to evaluate how this food matrix could influence the viability of the LAB strains. Surprisingly, all tested strains inoculated in the fresh cheese and exposed to low $\mathrm{pH}(2.5)$ for 3 $\mathrm{h}$ survived and maintained their high levels (Figure 1C and D). This result was also observed on the strains that presented the lowest survival rate when tested as washed or pure cultures (Figure $1 \mathrm{~B}$ ). Those strains that did not survive as pure cultures after $1 \mathrm{~h}$ at $\mathrm{pH} 2.5$ (e.g., strains L2A21R8 and L2B21R3) were considered as nonprobiotic. However, when inoculated in fresh cheese, they showed a survival rate around $100 \%$ after $3 \mathrm{~h}$ in the same conditions. These data show that fresh cheese greatly enhances the survival of LAB strains in simulated gastric juice, which can be attributed to the buffering capacity of the fresh cheese $(\mathrm{pH}$ around 6.5). Furthermore, the relatively dense matrix and fat content of this cheese may offer additional protection to LAB. In this way, all the LAB strains could be considered as resistant to gastric acidity when added to the manufacture of this cheese. Additionally, LAB were shown to grow well in this type of cheese, increasing at least $1 \log$ unit after $72 \mathrm{~h}$ of refrigeration (Coelho et al., 2014).

The resistance to bile salts and pancreatic enzymes were also tested in both pure cultures and fresh cheese environment (Figure 2). The majority of tested strains were found to be resistant to bile salts and pancreatin, even after $3 \mathrm{~h}$ of exposure, retaining their viability with negligible reduction in viable counts (Figure $2 \mathrm{~A}$ and $\mathrm{B}$ ). Few of the strains were severely affected by the presence of bile salts and pancreatin (strains L2B1K8 and L3B21K4, Figure 2B). However, when incorporated into fresh cheese, all strains presented a high survival rate (around 100\%) during the $3 \mathrm{~h}$ of incubation (Figure 2C and D). There are limited studies on the tolerance to simulated GIT conditions of probiotic cells inoculated in cheeses (Govender et al., 2014). Several authors have shown a relatively good support of the cheese matrix (cheddar, fresco cheese, whey cheese, and pasta filata) on bacterial survival at GIT conditions (Gardiner et al., 1999; Vinderola et al., 2000; Madureira et al., 2011; Pitino et al., 2012). However, a decrease of viable counts (around 2 to $>3$ log cycles) was generally observed in those studies. In opposition, no such decrease was observed in the present study, as all LAB strains tested showed a high survival rate when inoculated in Latin-style fresh cheese. This confirms this food matrix as an excellent way to protect LAB from the stress of the GIT environment (low pH in the stomach, bile salts and pancreatic enzymes in small intestine). Moreover, this type of fresh cheese provided greater protection to the LAB strains upon exposure to gastric juice and simulated intestinal conditions than matured cheeses or even yogurt (Gardiner et al., 1999; Pitino et al., 2012). The resistance to GIT conditions was checked by the in vitro tests generally used for the evaluation of the probiotic potential of a bacterial strain (Charteris et al., 1998; Collins et al., 1998; FAO/WHO, 2002). Although these assays are not fully adequate to predict the persistence of the strain in the human body, they are considered helpful in the selection of LAB strains for use as probiotics for human consumption (Schillinger et al., 2005).

The present study did not evaluate how the characteristic mild taste is affected by the inclusion of the LAB strains into this cheese type. Nevertheless, in a previous assay (Coelho et al., 2014), we investigated the sensory profiles of LAB-inoculated Latin-style fresh cheeses and found no significant differences in the sensory attributes tested. When strains with low acidifying capacities were used, Latin-style fresh cheeses were well accepted by consumers.

The protective effect of this cheese matrix was also tested against L. monocytogenes, as Latin-style fresh cheeses were particularly sensitive to colonization by this pathogen. Due to its high $\mathrm{pH}$, L. monocytogenes is able to survive and grow in fresh cheese, even at refrigeration temperatures (Coelho et al., 2014). Surprisingly, L. monocytogenes inoculated in fresh cheese did not survive under the low $\mathrm{pH}$ tested (Figure 3). These results suggested that the observed protective effect of cheese matrix on LAB is not exerted on other types of bacteria, such as L. monocytogenes. Moreover, the buffering capacity of the fresh cheese cannot be the only explanation for the high LAB survival rate observed in the simulated GIT conditions because, despite its buffering capacity, L. monocytogenes inocu- 

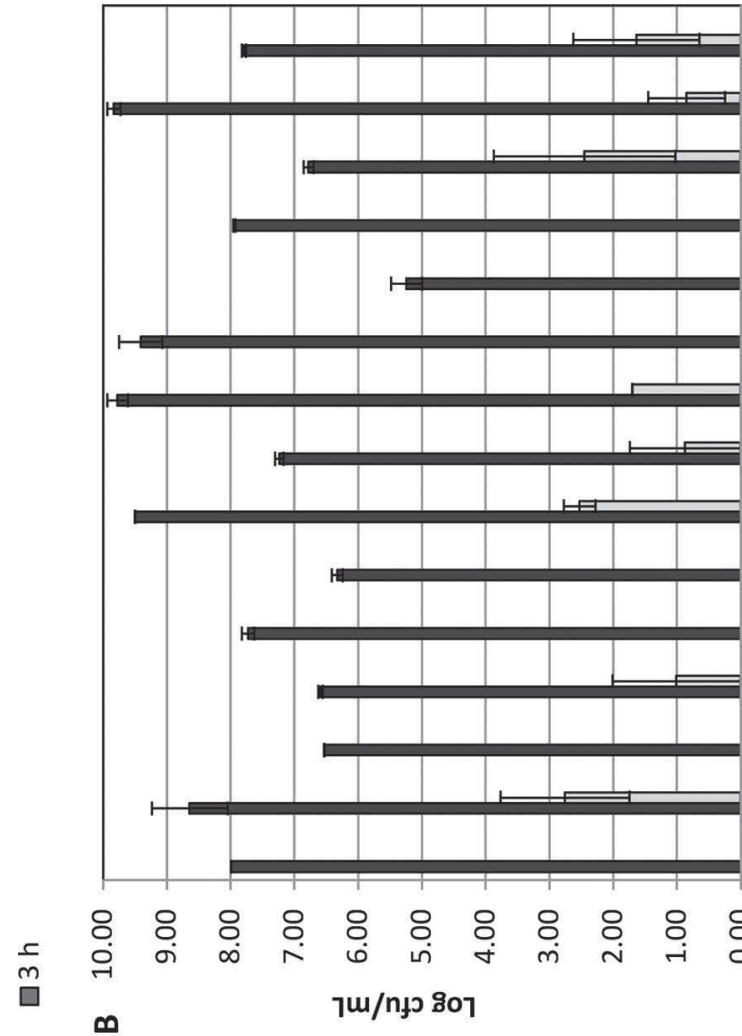

$\stackrel{\sim}{2}$

N

돈

$\infty$
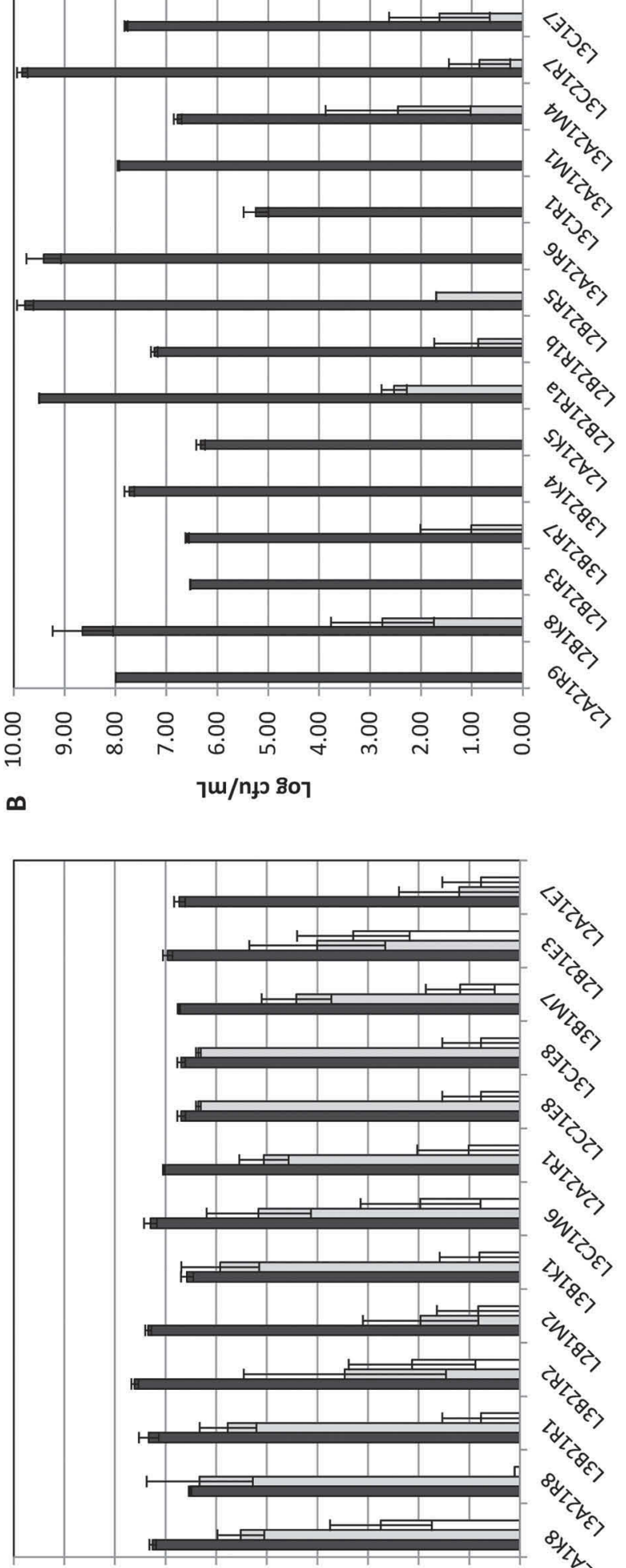

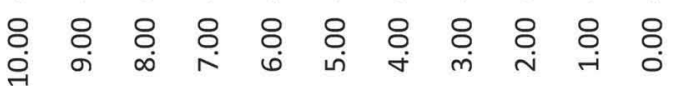

$\varangle$

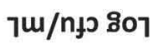

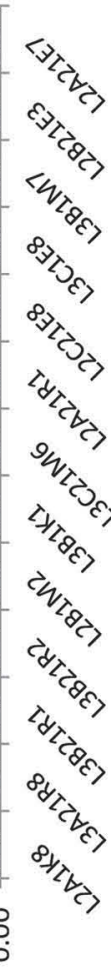

웅

$u$

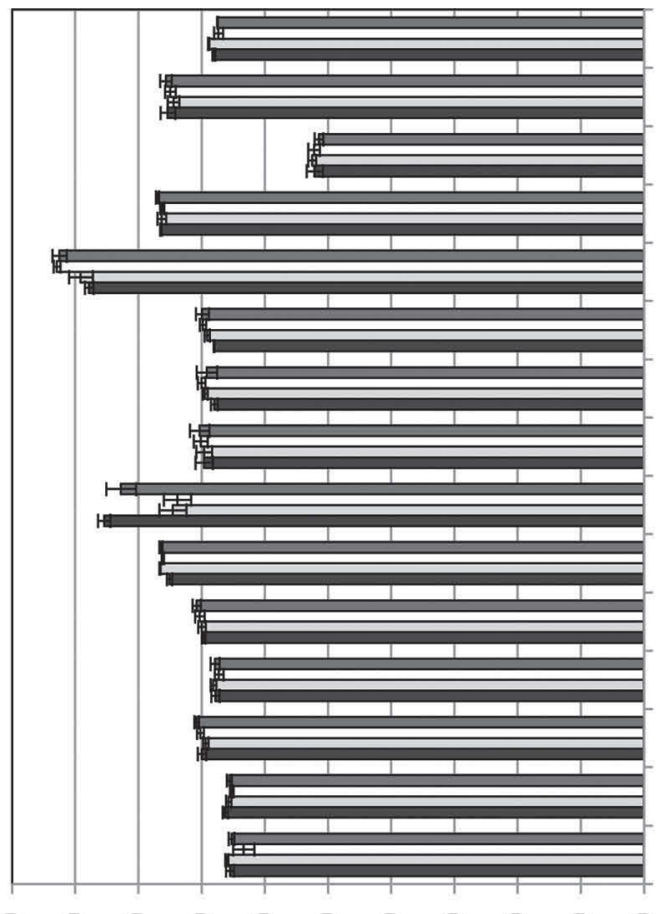

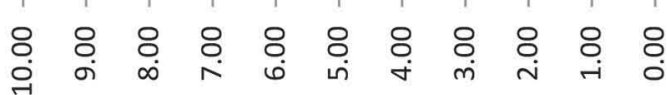

ךu/nృ ริ01

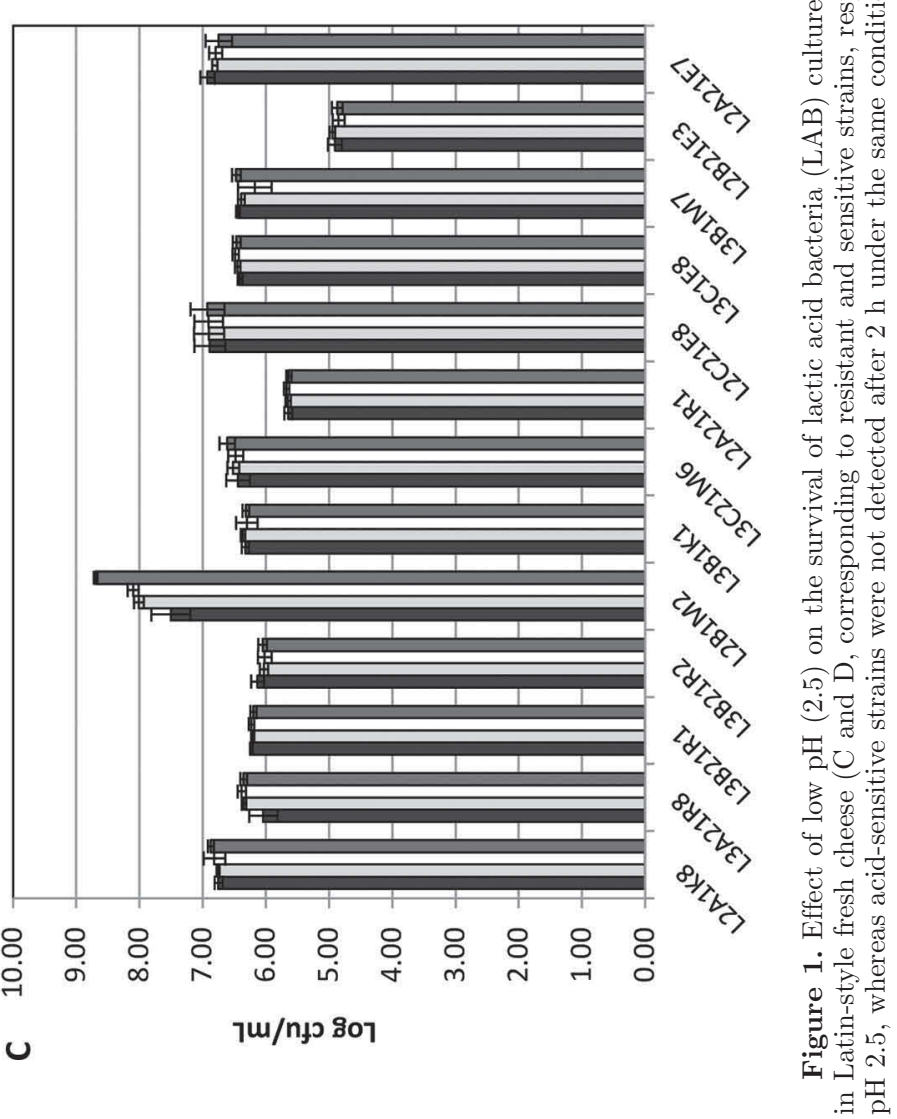



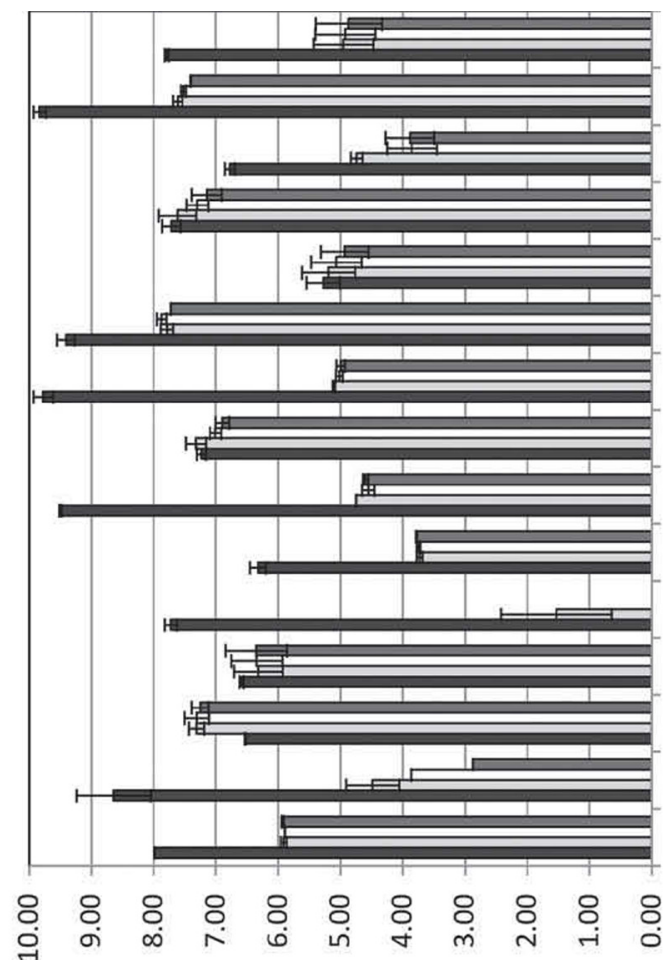

$\infty$

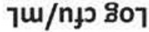

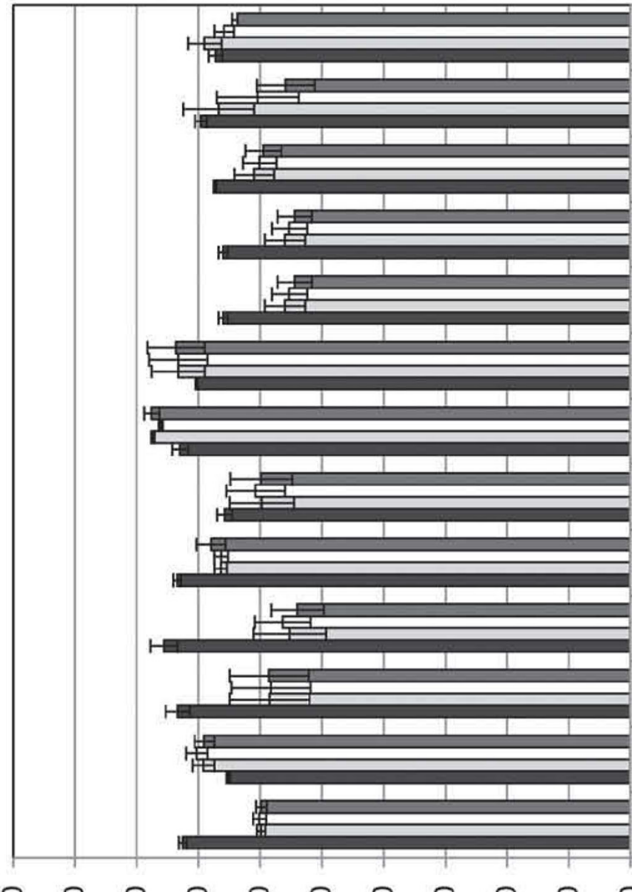

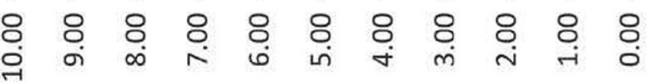

$\varangle$

ךய/nృ ริอา
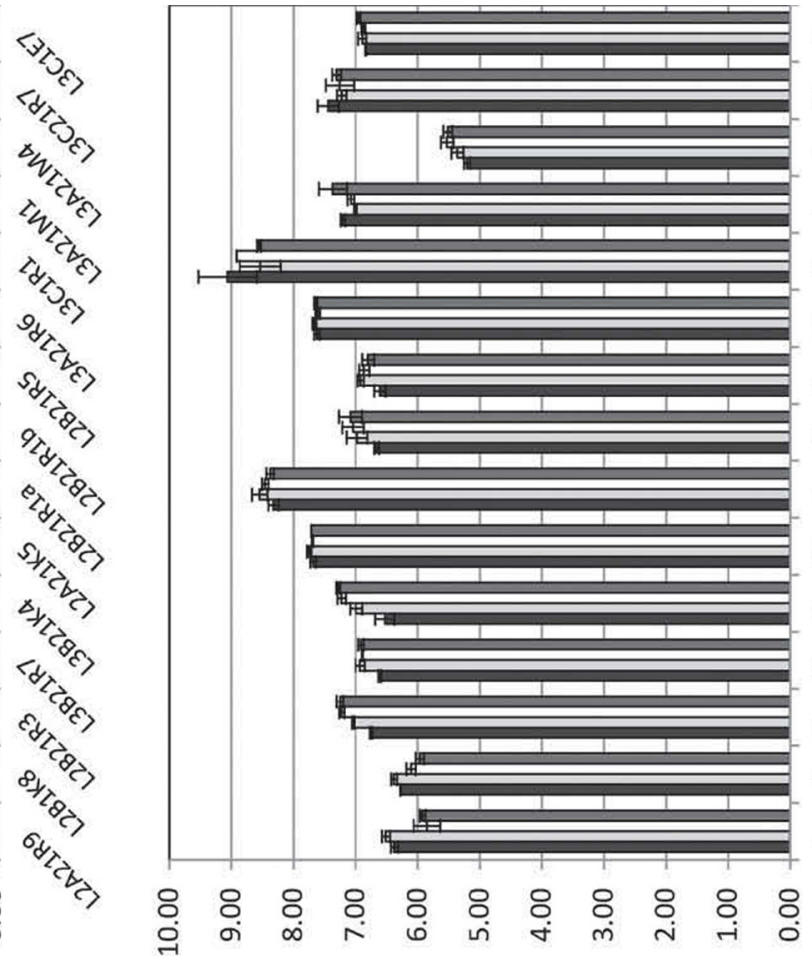

ךய/

อ

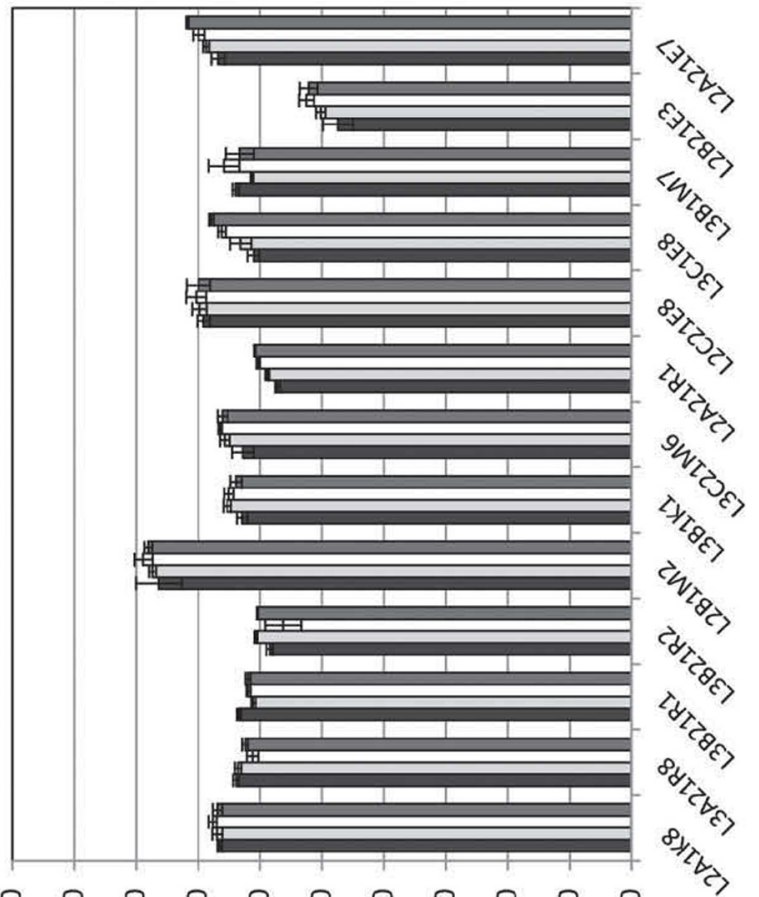

○

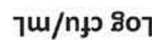

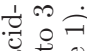

造

$\leq$

㟧.

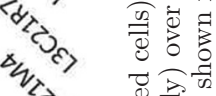

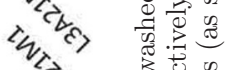

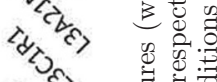

के

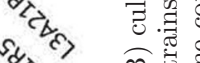

हि,

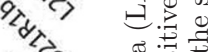

बis

s/ 20 ,

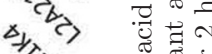

यें हो,

हो के

पे के बह

/

के के

㩆

$\circ$

证

U。

实

政

of

要

so

要通部

글

욛ำ

ช.

政

苞范

규.

ॠ

羊

की

蛋

ำ สี

웡

0 范

为运范

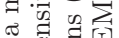

눙

记

丞藏

$i^{-1}$. के

0 00

$\exists$ 索

000 \%

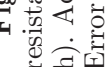




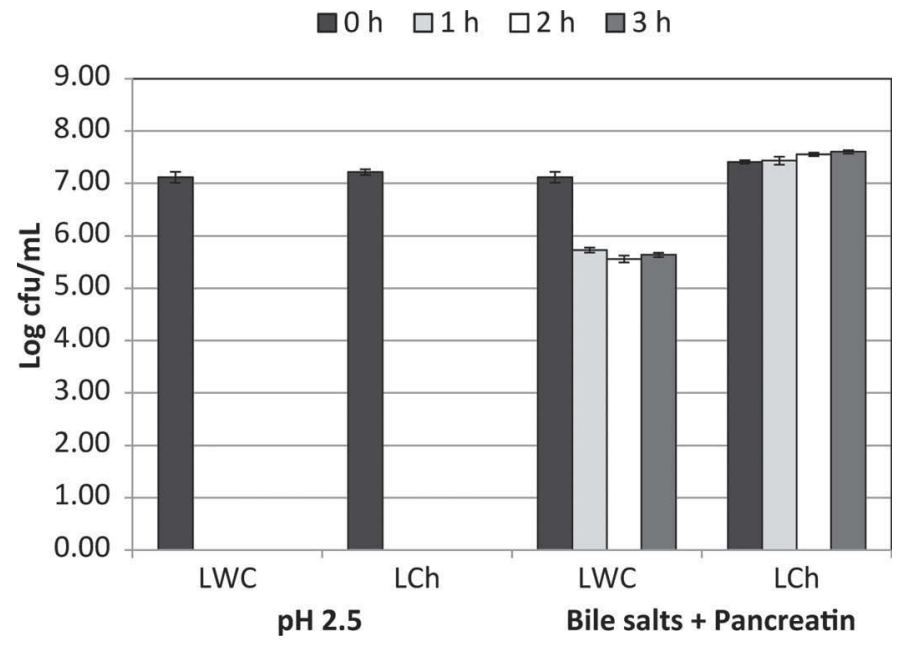

Figure 3. Effect of low pH (2.5) and a mixture of bile salts and pancreatin on Listeria monocytogenes washed cell (LWC) counts and L. monocytogenes inoculated in Latin-style fresh cheese $(\mathrm{LCh})$ over time $(0$ to $3 \mathrm{~h})$. Error bars represent SEM of 4 replicates.

lated in the cheese did not survive in the acidic conditions of the stomach after $1 \mathrm{~h}$. To our knowledge, this is the first time that such an effect was detected. These data suggest factors other than buffering capacity of fresh cheese that may increase the survival of the LAB strains, but not L. monocytogenes, when exposed to low $\mathrm{pH}$. This observation may also help to explain the current perception that, although food contaminated with L. monocytogenes may be frequently consumed by the public, it seldom results in listeriosis (Hitchins, 1996). Milk and dairy products have been implicated in the majority of the listeriosis outbreaks (PricopeCiolacu et al., 2013). Thus, it is possible that listeria numbers in dairy foods may be greatly reduced when submitted to the acidic conditions of the stomach for enough time. This hypothesis is also supported by the suggestion that antacid consumption is a predisposing factor for listeriosis (Hitchins, 1996). In contrast, $L$. monocytogenes was found to be resistant to bile salts and pancreatic enzymes in both pure culture and fresh cheese environment (Figure 3).

In conclusion, we demonstrated that the use of Latinstyle fresh cheese markedly enhances LAB survival rate to simulated GIT conditions. As this is an important factor for the selection of new probiotic candidates, this type of cheese may be a useful tool in improving the survival of probiotics through the human GIT. Moreover, due to the absence of a starter culture, LAB survive well during Latin-style fresh cheese manufacture and storage, making this product a good alternative for delivery of probiotics into the intestine. In addition, the protective effect of this cheese type to acidic $\mathrm{pH}$ was not observed with L. monocytogenes.

\section{ACKNOWLEDGMENTS}

This work was financed by National funds from Fundação para a Ciência e Tecnologia (FCT; Portugal), Project PTDC/AGR-ALI/104385/2008. The PhD grant of M. Domingos-Lopes was supported by Fundo Regional da Ciência e Tecnologia (FRCT; Portugal), project M3.1.2/F/009/2011.

\section{REFERENCES}

Burns, P., L. Lafferriere, G. Vinderola, and J. Reinheimer. 2014. Influence of dairy practices on the capacity of probiotic bacteria to overcome simulated gastric digestion. Int. J. Dairy Technol. $67: 448-457$.

Charteris, W. P., P. M. Kelly, L. Morelli, and J. K. Collins. 1998. Development and application of an in vitro methodology to determine the transit tolerance of potentially probiotic Lactobacillus and Bifidobacterium species in the upper human gastrointestinal tract. J. Appl. Microbiol. 84:759-768.

Coelho, M. C., C. C. G. Silva, S. C. Ribeiro, M. L. N. E. Dapkevicius, and H. J. D. Rosa. 2014. Control of Listeria monocytogenes in fresh cheese using protective lactic acid bacteria. Int. J. Food Microbiol. 191:53-59.

Collins, J. K., G. Thornton, and G. O. Sullivan. 1998. Selection of probiotic strains for human applications. Int. Dairy J. 8:487-490.

Cook, M. T., G. Tzortzis, D. Charalampopoulos, and V. V. Khutoryanskiy. 2012. Microencapsulation of probiotics for gastrointestinal delivery. J. Control. Release 162:56-67.

Corbo, M. R., A. Bevilacqua, L. Petruzzi, F. P. Casanova, and M. Sinigaglia. 2014. Functional beverages: The emerging side of functional foods commercial trends, research, and health implications. Compr. Rev. Food Sci. Food Saf. 13:1192-1206.

Evert-Arriagada, K., M. M. Hernandez-Herrero, J. J. GallardoChacon, B. Juan, and A. J. Trujillo. 2013. Effect of high pressure processing on volatile compound profile of a starter-free fresh cheese. Innov. Food Sci. Emerg. Technol. 19:73-78.

FAO/WHO. 2002. Guidelines for the Evaluation of Probiotics in Food. Food and Health Agricultural Organisation of the United Nations, Rome, Italy.

Faye, T., A. Tamburello, G. E. Vegarud, and S. Skeie. 2012. Survival of lactic acid bacteria from fermented milks in an in vitro digestion model exploiting sequential incubation in human gastric and duodenum juice. J. Dairy Sci. 95:558-566.

Fijan, S. 2014. Microorganisms with claimed probiotic properties: An overview of recent literature. Int. J. Environ. Res. Public Health 11:4745-4767.

Gardiner, G., C. Stanton, P. B. Lynch, J. K. Collins, G. Fitzgerald, and R. P. Ross. 1999. Evaluation of Cheddar cheese as a food carrier for delivery of a probiotic strain to the gastrointestinal tract. J. Dairy Sci. 82:1379-1387.

Govender, M., Y. E. Choonara, P. Kumar, L. C. du Toit, S. van Vuuren, and V. Pillay. 2014. A review of the advancements in probiotic delivery: Conventional vs. non-conventional formulations for intestinal flora supplementation. AAPS PharmSciTech 15:29-43.

Gregoret, V., M. J. Perezlindo, G. Vinderola, J. Reinheimer, and A. Binetti. 2013. A comprehensive approach to determine the probiotic potential of human-derived Lactobacillus for industrial use. Food Microbiol. 34:19-28.

Hitchins, A. D. 1996. Assessment of alimentary exposure to Listeria monocytogenes. Int. J. Food Microbiol. 30:71-85.

Kabuki, D. Y., A. Y. Kuaye, M. Wiedmann, and K. J. Boor. 2004. Molecular subtyping and tracking of Listeria monocytogenes in latinstyle fresh-cheese processing plants. J. Dairy Sci. 87:2803-2812.

Karimi, R., A. M. Mortazavian, and A. Amiri-Rigi. 2012. Selective enumeration of probiotic microorganisms in cheese. Food Microbiol. 29:1-9. 
Madureira, A. R., M. Amorim, A. M. Gomes, M. E. Pintado, and F. X. Malcata. 2011. Protective effect of whey cheese matrix on probiotic strains exposed to simulated gastrointestinal conditions. Food Res. Int. 44:465-470.

Monteagudo-Mera, A., L. Rodriguez-Aparicio, J. Rua, H. MartinezBlanco, N. Navasa, M. R. Garcia-Armesto, and M. A. Ferrero. 2012. In vitro evaluation of physiological probiotic properties of different lactic acid bacteria strains of dairy and human origin. J. Funct. Foods 4:531-541.

Nueno-Palop, C., and A. Narbad. 2011. Probiotic assessment of Enterococcus faecalis CP58 isolated from human gut. Int. J. Food Microbiol. 145:390-394.

Pitino, I., C. L. Randazzo, K. L. Cross, M. L. Parker, C. Bisignano, M. S. J. Wickham, G. Mandalari, and C. Caggia. 2012. Survival of Lactobacillus rhamnosus strains inoculated in cheese matrix during simulated human digestion. Food Microbiol. 31:57-63.

Pricope-Ciolacu, L., A. I. Nicolau, M. Wagner, and K. Rychli. 2013. The effect of milk components and storage conditions on the virulence of Listeria monocytogenes as determined by a Caco-2 cell assay. Int. J. Food Microbiol. 166:59-64.

Rehaiem, A., Z. Ben Belgacem, M. R. Edalatian, B. Martinez, A. Rodriguez, M. Manai, and N. P. Guerra. 2014. Assessment of potential probiotic properties and multiple bacteriocin encodinggenes of the technological performing strain Enterococcus faecium MMRA. Food Contr. 37:343-350.

Ribeiro, S. C., M. C. Coelho, S. D. Todorov, B. Franco, M. L. E. Dapkevicius, and C. C. G. Silva. 2014. Technological properties of bacteriocin-producing lactic acid bacteria isolated from Pico cheese an artisanal cow's milk cheese. J. Appl. Microbiol. 116:573-585.

Ritter, P., C. Kohler, and U. von Ah. 2009. Evaluation of the passage of Lactobacillus gasseri $\mathrm{K} 7$ and bifidobacteria from the stomach to intestines using a single reactor model. BMC Microbiol. 9:87.

Schillinger, U., C. Guigas, and W. H. Holzapfel. 2005. In vitro adherence and other properties of lactobacilli used in probiotic yoghurtlike products. Int. Dairy J. 15:1289-1297.

Sharp, M. D., D. J. McMahon, and J. R. Broadbent. 2008. Comparative evaluation of yogurt and low-fat Cheddar cheese as delivery media for probiotic Lactobacillus casei. J. Food Sci. 73:M375M377.

Vinderola, C. G., W. Prosello, D. Ghiberto, and J. A. Reinheimer. 2000. Viability of probiotic (Bifidobacterium, Lactobacillus acidophilus and Lactobacillus casei) and nonprobiotic microflora in Argentinian Fresco cheese. J. Dairy Sci. 83:1905-1911. 